\title{
Distribution of moisture in reconstructed oil paintings on canvas during absorption and drying: a neutron radiography and NMR study
}

\author{
Roel Hendrickx¹, Ester S.B. Ferreira1,7, Jaap J. Boon¹, Guylaine Desmarais², Dominique Derome ${ }^{3}$, Lora Angelova ${ }^{4}$, David \\ Mannes $^{5}$, Anders Kaestner ${ }^{5}$, Henk (H.P.) Huinink ${ }^{6}$, Kees (C.J.) Kuijpers ${ }^{6}$, Benjamin Voogt ${ }^{6}$, and Emma Richardson ${ }^{4}$ \\ ${ }^{1}$ Department of Art Technology, Swiss Institute for Art Research, Zollikerstrasse 32, 8032 Zurich, Switzerland \\ ${ }^{2}$ Chair of Building Physics, ETH Zürich, Stefano-Franscini-Platz 5, 8093 Zürich Hönggerberg, Switzerland \\ ${ }^{3}$ Multiscale Studies in Building Physics, Empa Swiss Federal Laboratories for Materials Science and Technology, \\ Überlandstrasse 129, 8600 Dübendorf, Switzerland \\ ${ }^{4}$ Department of History of Arts, University College London, Gower Street, London, WC1E 6BT, United Kingdom \\ ${ }^{5}$ Neutron Imaging and Activation Group, Paul Scherrer Institute, 5232 Villigen, Switzerland \\ ${ }^{6}$ Eindhoven University of Technology, Department of Applied Physics, Den Dolech 2, 5612 AZ Eindhoven P.O. Box 513, 5600 \\ MB Eindhoven, the Netherlands \\ ${ }^{7}$ CICS Cologne Institute for Conservation Sciences, Technische Hochschule Köln, University of Applied Sciences \\ Campus Südstadt, Ubierring 40, 50678 Köln, Germany
}

Correspondence to: Roel Hendrickx, Department of Art Technology, Swiss Institute for Art Research, Zollikerstrasse 32 , 8032 Zurich, Switzerland. Email: roel.hendrickx@sik-isea.ch

\begin{abstract}
Moisture is a driving factor in the long-term mechanical deterioration of canvas paintings, as well as for a number of physico-chemical degradation processes. Since the 1990s a number of publications have addressed the equilibrium hygroscopic uptake and the hygro-mechanical deformation of linen canvas, oil paint, animal glue, and ground paint. In order to visualise and quantify the dynamic behaviour of these materials combined in a painting mock-up or reconstruction, we have performed custom-designed experiments with neutron radiography and nuclear magnetic resonance (NMR) imaging. This paper reports how both techniques were used to obtain spatially and temporally resolved information on moisture content, during alternate exposure to high and low relative humidity, or in contact with liquids of varying water activities.
\end{abstract}

We observed how the canvas, which is the dominant component in terms of volumetric moisture uptake, absorbs and dries rapidly, and, due to its low vapour resistance, allows for vapour transfer towards the ground layer. Moisture desorption was generally found to be faster than absorption. The presence of sizing glue leads to a local increase of moisture content. It was observed that lining a painting with an extra canvas results in a damping effect: i.e. absorption and drying are significantly slowed-down. The results obtained by NMR are complementary to neutron radiography in that they allow accurate monitoring of water ingress in contact with a liquid reservoir. Quantitative results are in good agreement with adsorption isotherms. The findings can be used for risk analysis of paintings exposed to changing micro-climates or subjected to conservation treatments using water. Future studies addressing moisture-driven deformation of paintings can make use of the proposed experimental techniques.

This document is the accepted manuscript version of the following article: Hendrickx, R., Ferreira, E. S. B., Boon, J. J., Desmarais, G., Derome, D., Angelova, L., Mannes, D., Kaestner, A., Huinink, H. H. P., Kuijpers, K. C. J., Voogt, B., \& 1 Richardson, E. (2017). Distribution of moisture in reconstructed oil paintings on canvas during absorption and drying: a neutron radiography and NMR study. Studies in Conservation, 62(7), 393-409. https://doi.org/10.1080/00393630.2016.1181899 


\section{Introduction}

The interaction of cultural heritage objects with moisture is an important factor to consider in any risk analysis (Michalski 1991). The link between the measured microclimate of an object and the risk of damage is usually far from evident, in particular in the case of artefacts of complex composition. This knowledge is, nevertheless, essential in order to set target values for the microclimate of a museum, values which are safe, yet do not require a high use of primary energy for climate control (Michalski, 2007, Brophy and Wylie, 2013). Some researchers have endeavoured to make these links for objects made of wood using physical models (Huijbregts, et al. 2012, Bertolin \& Camuffo, 2015). However, canvas paintings are notoriously difficult to study: a straightforward approach based on simple stress-strain curves, equilibrium isotherms, and hygric dilatation curves cannot capture the full complexity they display. The diverse compositional materials of paintings have been the subject of extensive studies of hygro-mechanical deformation (Mecklenburg \& Tumosa, 1991; Young, 1996). Limits of relative humidity $(\mathrm{RH})$ have been proposed within which fluctuations have been deemed safe for each individual component (linen, ground, paint, glue) (Mecklenburg, 2007). These studies and others have identified the differences in the properties of painting materials as the critical factor leading to differential deformation, internal stress and eventually cracking or cupping (Hedley, 1993).

Apart from hygro-mechanical phenomena, physico-chemical processes such as the efflorescence of soluble matter or the formation and migration of metal soaps are promoted by the presence of moisture, either as a reagent or as a catalyst (Ordonez and Twilley, 1998; Ferreira, et al., 2011; Van Loon, et al., 2012). Many conservation treatments involve the exposure of paintings to moisture for variable lengths of time. In order to control the potential risk during such treatments, and to have a better understanding of the interaction of painting components with ambient moisture, new experimental approaches were developed which allow for the elucidation of the moisture distribution under different conditions in individual layers.

Many of the established strategies for measuring moisture transport are either bulk methods - providing information about the whole volume of the sample - or equilibrium methods - providing information about a final condition or a stationary process. For example: in classical vapour diffusion tests, a membrane forms a vapour resistance between a low-RH and a high- $\mathrm{RH}$ environment, and the measured permeance is the result of the transport of moisture over the whole thickness of the membrane, plus surface phenomena on both sides. Knowing that the properties of the membrane depend on its moisture content, calculations, extra experiments and some substantiated assumptions are necessary to determine the quantity of moisture the membrane contains at a specific location and time and, from this, its permeability as a function of the moisture content (Hendrickx, et al., 2016). To overcome such difficulties, it can be helpful to use experimental methods that give spatial and temporal information of moisture content simultaneously. Both nuclear magnetic resonance (NMR) imaging and neutron radiography can measure a 1D or 2D distribution of moisture over a sample, at different spatial, temporal and moisture content resolutions. Along with X-ray radiography, these two techniques are currently among the most established methods for monitoring moisture transport. While X-ray radiography is not very sensitive and is primarily useful for the measurement of capillary absorption and high volumetric moisture contents (Roels \& Carmeliet, 2006), both neutron radiography and NMR imaging are highly sensitive, with detection limits for moisture content of 5 vol\% or less (Reuvers, 2012; Perfect, et al., 2014).

The use of neutron radiography as a quantitative method to study moisture absorption in paintings was explored recently (Boon, J.J., et al., 2015). Although control of the ambient conditions in the beamline and the precise alignment of samples are not straightforward, the authors show that it is feasible to obtain sufficient information for monitoring the temporally and spatially resolved moisture absorption in painting canvas with a ground layer. NMR methods for the monitoring of 
water transport in multilayer coatings have been developed for the study of modern car paint systems (Baukh, et al., 2012). Recently, a portable NMR device was successfully used to measure water uptake from aqueous cleaning gels in acrylic paint (Angelova, et al., 2016). Such portable devices can be used on-site or in a restoration workshop to monitor ongoing processes and have proven useful in a variety of studies related to cultural heritage (Blümich, et al., 2008; Capitani \& Di Tullio, 2012).

This paper aims at visualising and quantifying the spatial and temporal distribution of moisture in layered paintings with these two techniques using novel set-ups and following innovative experimental procedures. The study was undertaken within the framework of the art-historical investigations on the Swiss painter Cuno Amiet (1868-1961), whose work was investigated at the Swiss Institute for Art Research (Beltinger, et al., 2015). Using materials found to be representative of his early oeuvre and, in a broader sense, of Western European paintings from the time period around 1900, 'painting reconstructions' or 'mock-up paintings' were prepared for use in laboratory experiments. The chosen materials include several types of linen canvas, animal glue sizing, chalk-glue ground, and oil paint. Three different set-ups for neutron radiography are introduced, as well as the various methods used to process and analyse the data. The experiments were carried out on mock-up paintings with ground and oil paint, prepared on different types of sized canvas, as well as on separate samples of sized canvas alone. The latter allows for discerning between different methods of glue size application, which is relevant because of the high moisture sorption potential of animal glue. Furthermore, the effect of lining a painting on its moisture response was investigated using two types of lining canvas. An experiment carried out with the painting sample placed perpendicularly to the neutron beam is also presented for the visualisation of moisture distribution over the plane of the canvas.

The NMR studies presented cover several additional aspects: absorption in the isolated paint layers, a process which is difficult to capture with neutron radiography, and the uptake of moisture through the oil paint layer, (i.e. the layer with the lowest permeability). To this end, the samples were exposed to a reservoir of bulk water on top of the oil paint layer. Though this situation is not representative of storage or conservation conditions (and only likely in the event of a flood), such experiments allow for the determination of diffusion coefficients of water in oil paint and - when continued until saturation - of the equilibrium moisture content at a given water activity. Water from the underlying canvas and ground layers may permeate into the paint layers, enabling subsequent chemical and physical processes involved in a variety of degradation pathways. Whereas the neutron radiography experiments result in a $2 \mathrm{D}$ intensity distribution map, the NMR studies provide information about the molecular mobility in the materials by measuring the so-called $\mathrm{T}_{2}$ relaxation time at various spatial locations within the samples. Information from the relaxation times can be used to determine the position of the edges of the sample, providing a method to quantify swelling in a reliable way.

\section{Materials and methods}

\section{Production of reconstructed painting and NMR samples}

Samples were produced using new materials and according to traditional methods which are relevant for the late $19^{\text {th }}$ and early $20^{\text {th }}$ century work of Cuno Amiet (1868-1961) (Carlyle, 2012; Beltinger, et al., 2015). Two reconstructed painting samples were prepared, consisting of the following consecutive layers: linen canvas, glue sizing applied in the form of a liquid or a gel, ground paint consisting of 1 part of chalk powder with 1 part of glue by mass, and oil paint composed of brown umber pigment in linseed oil (samples 1-2, Table 1). The gel sized canvas reconstruction was further lined by two types of lining canvas (samples 3-4, see below for specifications). Three types of sized canvas without ground or paint were prepared separately (samples 5-7). The canvases used for these samples are all traditional linen canvases, but they have 
diverse weave parameters. Linen canvas types 1 to 3 have similar yarns counts in warp and weft $(16 \times 15,19 \times 18$, and $17 \times$ 15 respectively) and they weigh 371,337 , and $356 \mathrm{~g} / \mathrm{m}^{2}$. Linen canvas type 4 has a different weave: the yarns are spaced from each other, leaving an open, mesh-like structure with $11.5 \times 12$ yarns $/ \mathrm{cm}$; the weight is $160 \mathrm{~g} / \mathrm{m}^{2}$. The detailed procedures of the preparation and specifications of the materials can be found elsewhere (Hendrickx, et al., 2016); for canvas type 4 and the glue used for its sizing (sample 7, Table 1): see Carlyle, 2013.

Table 1. List of tested samples. Thicknesses are based on microscopic images of cross sections (samples 1-4) or a digital thickness gauge (sample 8).

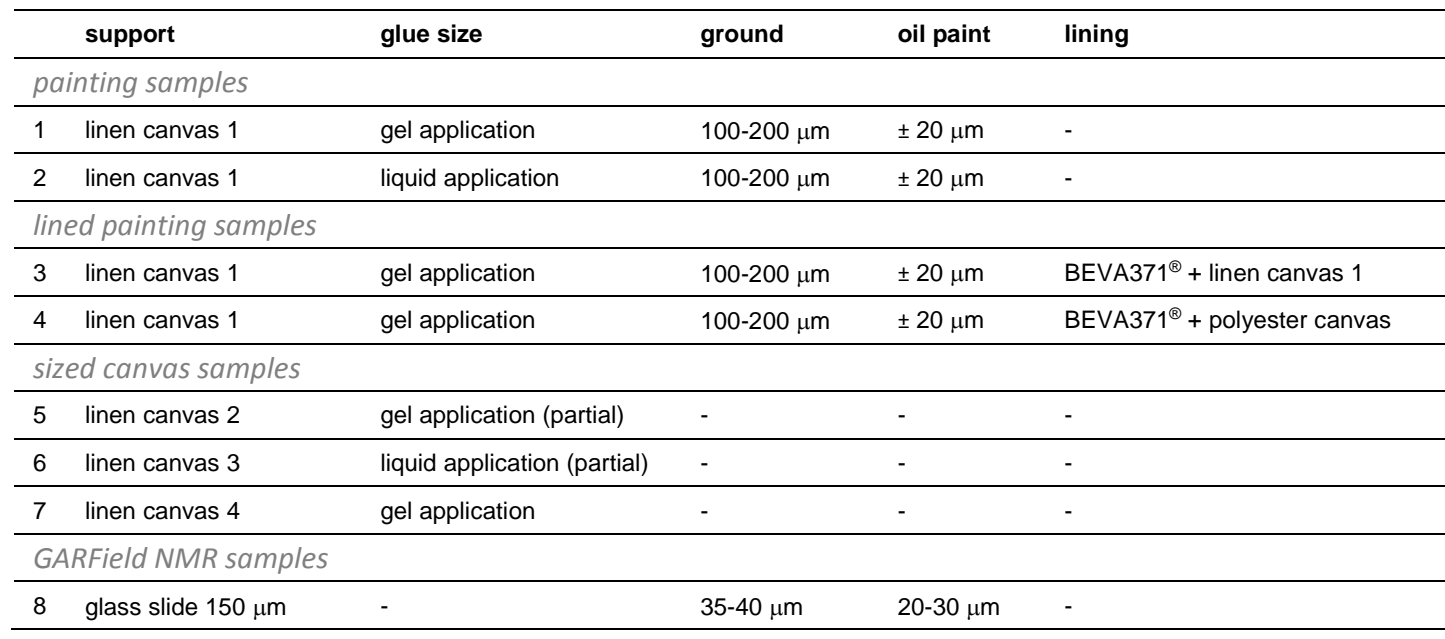

The attachment of a second canvas to reinforce a weakened original canvas of a painting is known as lining and was common practice until the last quarter of the $20^{\text {th }}$ century (Hackney, et al., 2012). Because lined paintings are expected to have a different moisture absorption behaviour relative to unlined paintings, two additional samples have been included in this study: a mock-up with a linen lining canvas identical to that used in the original painting mock-up, and a second mockup lined with P110 Lascaux polyester canvas (see Young \& Jardine (2012) for a description of the morphological and hygromechanical properties of P110). In both cases a $25 \mu \mathrm{m}$ BEVA371 ${ }^{\circledR}$ glue film was used as a binding agent (Berger, 1972). This glue material was selected because it is well documented and has been extensively used by conservators during the last three decades. Because of the limited size of the samples, the lining was applied by hand using a flat iron, and not with a heated vacuum table typically used for larger interventions. The film was first attached to the lining canvas using a protective sheet and then this assembly was joined to the back of the painted canvas, at a temperature of $65 \pm 3{ }^{\circ} \mathrm{C}$ (monitored during lining with flat thermometer).

Linen canvas and animal glue have high moisture capacity relative to those of ground and oil paint (Figure 1). The chalkcontaining ground absorbs significantly less moisture than the glue it is prepared from, due to the very low volume fraction of the glue relative to the non-hygroscopic chalk particles. The oil paint layer is identified as the only layer with a significant vapour resistance (Figure 2). The value for vapour resistance is expressed here as the thickness, $L$, of an equivalent column of air which has the same vapour resistance, $R^{*}$, knowing that $1 \mathrm{~cm}$ of air has a resistance of $5.12 \cdot 10^{7} \mathrm{~m}^{2} \mathrm{sPa} / \mathrm{kg}$.

$$
g_{\text {diff }}=\frac{\Delta p_{v}}{R^{*}}, L=\frac{R_{\text {measured }}^{*}}{R_{1 \text { cm air }}^{*}}
$$

In this equation $g_{\text {diff }}$ stands for the diffusive vapour flux through the material, and $\Delta p_{v}$ for the difference in vapour pressure on either side. A continuous glue film has high vapour resistance, but in the case of a glue size applied to a painting canvas, the glue is always dispersed so that it cannot actually form a strong barrier. Depending on how the glue is distributed 

extra vapour resistance to the canvas. The low vapour resistance of the ground is due to its open porous microstructure.

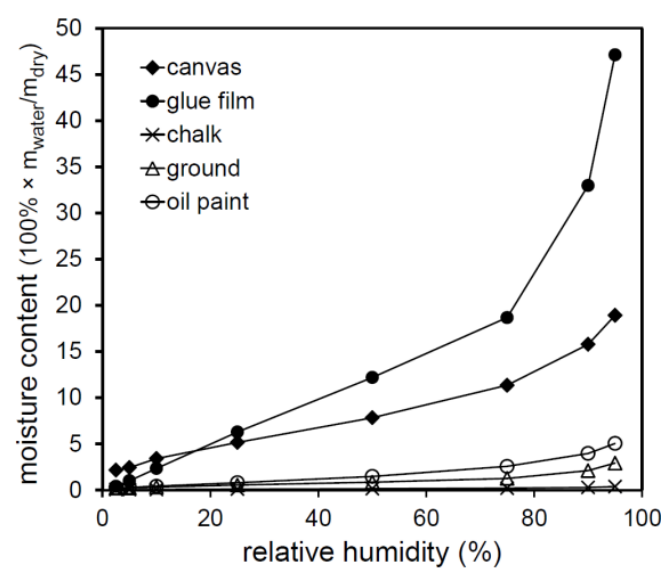

Figure 1. Adsorption isotherms of individual materials obtained by Dynamic Vapour Sorption (DVS) measurements (Hendrickx et al. 2016). Moisture content is expressed as a percentage of absorbed moisture mass relative to the dry mass of the material.

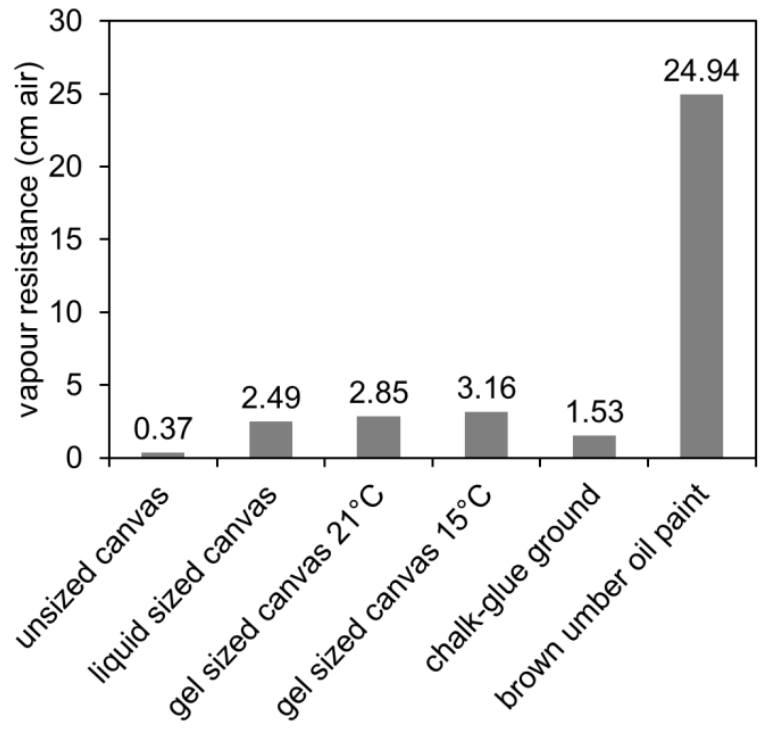

Figure 2. Vapour resistance of layers that form a reconstructed painting, expressed in $\mathrm{cm}$ of an air layer which has the same vapour resistance. Results obtained in a dry cup test at an average $\mathrm{RH}$ of 25\% (Hendrickx et al. 2016).

For NMR tests on isolated paint layers, a layer of chalk-glue ground was applied on a $150 \mu \mathrm{m}$ thick glass slide with a wet thickness of $120 \mu \mathrm{m}$ using a calibrated film applicator (Erichsen, Germany). After a drying time of at least 4 days, an oil paint layer of thickness $60 \mu \mathrm{m}$ was applied perpendicularly over the ground layer in the same manner. The samples were cured for 75 to 80 days (until the day of testing) in lab conditions, with moderate exposure to daylight. The final thickness of the ground layer reduced to 35-40 $\mu \mathrm{m}$ due to drying, while the oil paint layer had a thickness of 20-30 $\mu \mathrm{m}$. Thickness values for one layer were obtained by measuring a series of points with and without the layer in question using a digital thickness gauge.

\section{Neutron radiography}

In contrast to X-rays, the attenuation of a neutron beam is not dependent on atomic number, but rather on the nature of the nucleus. The strong interaction between neutrons and the nucleus of hydrogen makes neutron radiography an interesting tool to visualise water and light organic materials (Perfect, et al., 2014). The experiments were carried out at the ICON (Imaging with Cold Neutrons) beamline of the Paul Scherrer Institute in Villigen (CH) (Kaestner, et al., 2011). The beamline works with neutrons in the cold (i.e. low energy) spectrum, generated in the neutron spallation source SINQ (Blau, et al., 2009). As detector, a scintillator-CCD camera system described in detail by Lehmann et al. (2007) was used. The scintillator, which converts the incoming neutrons into visible light, was a Gadox $\left(\mathrm{Gd}_{2} \mathrm{O}_{2} \mathrm{~S}\right)$-screen with $20 \mu \mathrm{m}$ layer thickness. The detector provides a field of view of $27.6 \times 27.6 \mathrm{~mm}$, which is projected via a 1:1 lens onto a CCD camera with $2048 \times 2048$ pixels resulting in a pixel size of $13.5 \mu \mathrm{m}$. The actual spatial resolution with this set-up corresponds to 18 line pairs per mm (Kaestner, et al., 2011). This paper reports the results obtained during 3 experimental campaigns at ICON performed in 2011 and 2013 using set-up A (Figure 3) and 2014 using set-ups B and C (Figure 4).

Set-up A consists of a Teflon holder in which a sample is laid flat between two perforated Teflon plates (Boon, J.J., et al. 2015). This holder is screwed on top of an aluminium base, in which water can be injected with a remote-controlled syringe, so that the environment below the sample reaches an $\mathrm{RH}$ of $98-100 \%$ within a few minutes. Depending on the permeability of the sample and the seal at the top, the $\mathrm{RH}$ above the sample reaches $85-100 \% \mathrm{RH}$ within 30 to 100 minutes. The test 


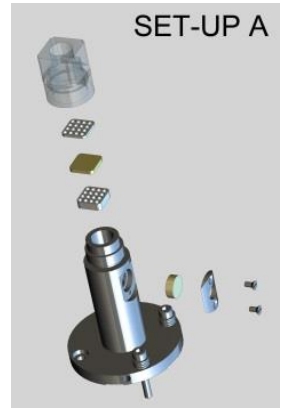

procedures consist of absorption of vapour during several hours, starting with a sample in relatively dry conditions (in desiccator at $15 \% \mathrm{RH}$, followed by exposure to ambient conditions in the beamline bunker for installation (during 1 to 10 min) until the start of the test, at 25 to $35^{\circ} \mathrm{C}$ and 25 to $\left.30 \% \mathrm{RH}\right)$. Neutron images were obtained every 103 seconds. The rough images were processed to remove outliers (ImageJ 1.49), corrected for detector-related errors (dark current) and unwanted radiation by backscattered neutrons in the beamline (black body correction), normalised to the open beam image, and segmented by thresholding (Matlab 8.3) (Figure 3c). The moisture distribution was obtained by computing the logarithm of the signal intensity ratio between the dry reference image and the time series of wet images. Calibration to determine the effective attenuation coefficient was based on gravimetric measurement results obtained during replicated experiments, performed after the beamline session (Boon, J.J., et al., 2015). Samples 5-7 were tested according to this procedure.
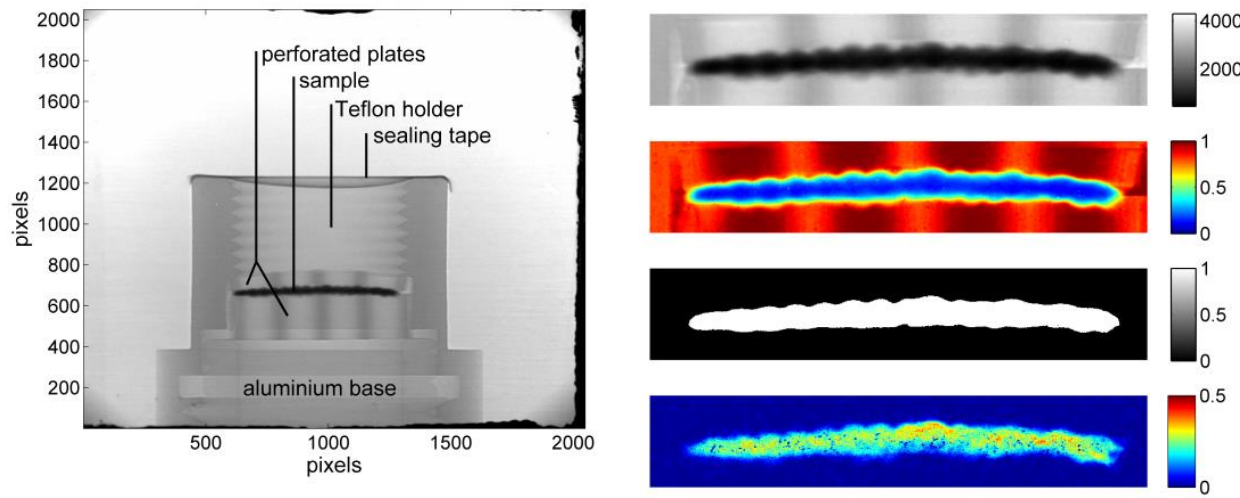

Figure 3. Overview of geometry and image operations for set-up A. Left: isometric view of the aluminium base (in black) and successive parts in Teflon, with the sample in brown. Centre: raw image (after corrections with dark current and black body images) showing the position of the sample in the holder as it is in the beamline. Right: from top to bottom: (top 1) raw image, (2) image normalised to open beam image, (3) segmented image with the object in white, and (bottom 4) logarithmic difference of wet and dry images at the end of the absorption phase. The sample shown here is sample 5, its left half being unsized, its right half having gel sizing.

During the 2014 campaign, another miniature climatic chamber was used, allowing the control of RH between 10 and $95 \%$, so that both absorption and desorption could be imaged. Two different types of sample holder allow imaging along the plane of the specimen (set-up B, Figure 4 left) and normal to it (set-up C, Figure 4 right).
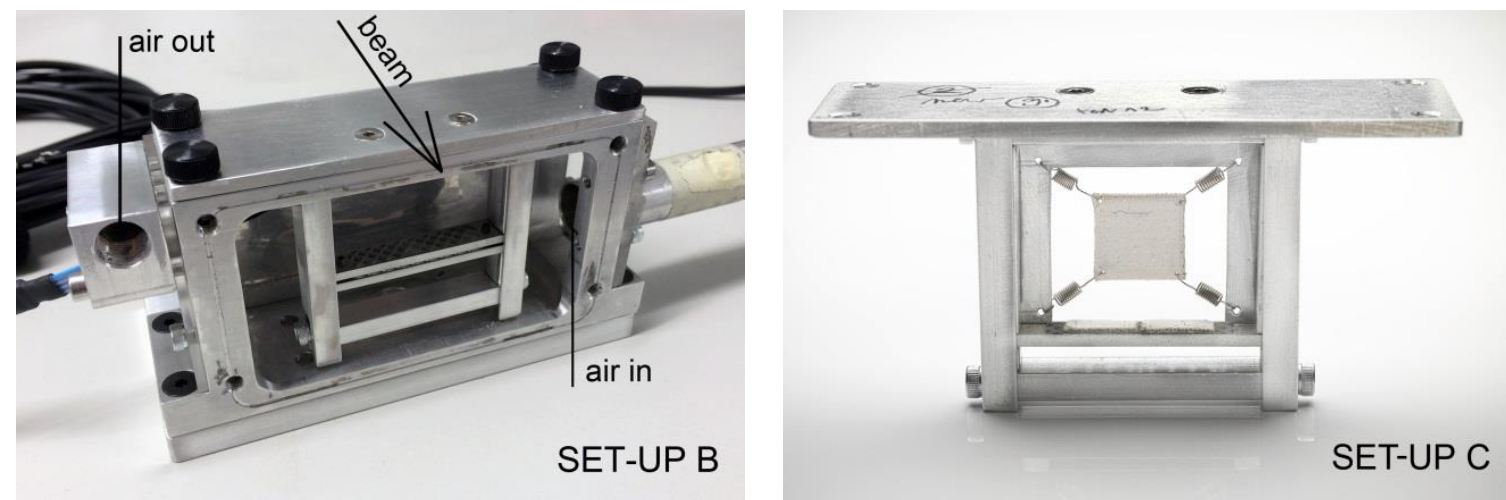

Figure 4. Climatic chamber for set-ups B and C as used in the neutron beamline during the 2014 session. Conditioned air comes in from the right side and is evacuated on the opposite side. The RH and temperature of the air was measured with sensors just after the inlet, close to the sample, and just before the outlet. Left: chamber with front panel taken off, showing the sample holder of set-up B, which forms one piece with the top cover and contains two samples in flat position in between perforated plates. The whole set-up is made of aluminium, which is nearly transparent to neutrons. Right: Set-up C with frontal sample holder, taken out of the climatic chamber: one square sample is suspended between springs and two flat samples are laid freely on a perforated plate, one with the warp direction of the canvas weave along the beam direction and the other with the weft direction along the beam direction.

When measuring in set-up $B$, the detector was tilted to an angle of $80^{\circ}$ resulting in an increase of the vertical resolution by a factor of 5.76 (Boillat, et al., 2010 and Figure 5). In the detector image, the wavy edge on the bottom of the samples which 
reflects the shape of the woven canvas is strongly stretched (note the different scale bars in both directions). The decreased neutron flux reaching the tilted detector necessitates a longer exposure time to compensate, so that the time between two measurements increases to 2.15 minutes. The obtained signal to noise ratio is also lower; to correct this, extra image enhancement steps were added to the procedure. The full data processing routine is as follows:

1. Correction for bias introduced by the CCD camera detector (using a dark current image) and for unwanted scattering in the beamline (using a black body image) (Matlab);

2. Binning, i.e. merging of $2 \times 2$ pixels to form 1 superpixel having the median value, using the standard plug-in in Imagej;

3. $3 \mathrm{D}$ median filtering with radius 3 pixels in all directions, the $3^{\text {rd }}$ dimension being time (the second and penultimate images have radius 2 pixels in the direction time and the first and last image are filtered flat, i.e. only over the dimensions of the single image) (ImageJ (Ollion et al. 2013))

4. Normalisation to an open beam image (Matlab);

5. Registration of the whole time series to the first image, i.e. all images are transformed using a combination of a translation, rotation and scaling, so that the objects have an optimal overlap with the first (dry reference) image. This is necessary to compensate for swelling and movement of the samples during the test, in order to enable us to make the pixelwise subtractions between different images. The software used is the plugin Stackreg in ImageJ (Thévenaz et al. 1998).

6. Segmentation of both registered and non-registered time-series by thresholding of the images, followed by a morphological opening (erosion followed by dilation) using a disk of radius 2 pixels as the structural element (Matlab);

7. Calculation of the moisture content distribution for semi-quantitative analysis; calibration using gravimetric data for quantitative analysis.

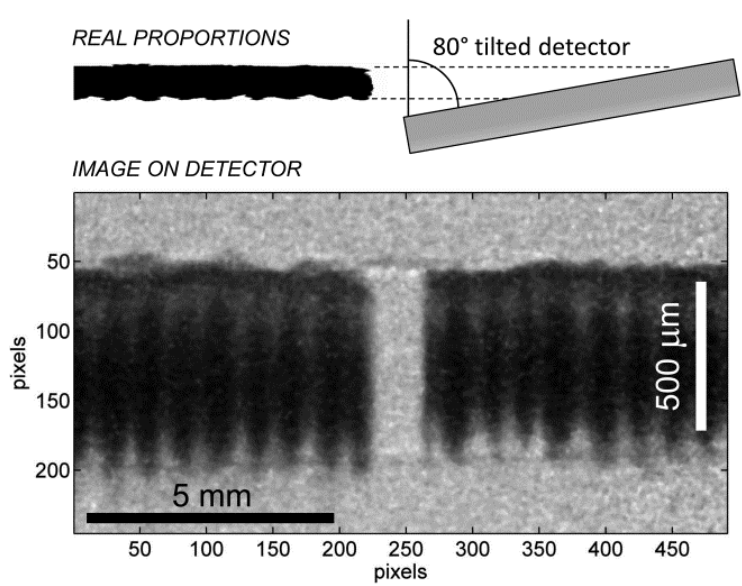

Figure 5. Top: Schematic representation of the increased vertical resolution obtained by tilting the detector. Bottom: The resulting image on the detector is stretched in the vertical direction by a factor $1 / \cos \left(80^{\circ}\right)=5.76$.

The experimental procedure consisted of a water vapour absorption stage of 3 to $4 \mathrm{hrs}$ at $90 \pm 3 \% \mathrm{RH}$ starting from dry condition, followed by a 3-hour stage of desorption at $15 \pm 3 \% \mathrm{RH}$. Calibration of the effective attenuation coefficient was carried out using the results of gravimetric measurements performed at the beamline before the test and at the end of the wetting phase with a $0.1 \mathrm{mg}$ resolution precision balance. Samples 1-4 were measured according to this procedure. 


\section{NMR imaging}

The magnetic properties of the nucleus of hydrogen make it possible to use Nuclear Magnetic Resonance relaxometry for obtaining information on the spatial distribution of moisture in materials. With this technique, the sample is subjected to a strong magnetic field, $B$, which induces a magnetisation aligned parallel to the magnetic field. Using a radio frequency pulse, a second magnetic field, $B_{1}$, is introduced perpendicularly to $B$, which can flip the magnetisation of the nuclei away from the equilibrium direction. To that end a radiofrequency (RF) signal with a frequency of $\gamma B$ has to be applied, where $\gamma$ is the gyromagnetic ratio of a nucleus. In NMR imaging the magnetic field is made position-dependent and as result nuclei located in different positions in space resonate with different frequencies. The NMR imaging methods used in this paper make use of the constant gradient $G$ in the field in one direction.

$$
B=B_{0}+G x
$$

After exciting the nuclei, a detectable signal is obtained which decays in intensity over time. This process is characterised by two relaxation times: the transverse $\left(T_{2}\right)$ and longitudinal $\left(T_{1}\right)$ relaxation times. The signal strength depends on the density of $\mathrm{H}$ nuclei, and the relaxation time depends on the mobility of the molecules (self-diffusion of the water molecules and interaction with other dipoles) and heterogeneity of the magnetic field. Using a well-defined series of radiofrequency pulses, called a CPMG sequence $\left(90^{\circ}, 180^{\circ}, 180^{\circ}, 180^{\circ}, \ldots\right)$, one can accurately measure $T_{2}$ as well as $T_{1}$. The CPMG sequence was used in two different set-ups: the portable NMR Mouse PM-5, an apparatus designed and commercialised by Magritek (Aachen, Germany), and a GARField NMR apparatus.

\section{NMR Mouse PM-5}

The NMR Mouse uses a $B_{0}$ field that is created by an arrangement of 4 permanent magnets (Blümich, et al., 2008). The field, directed horizontally when the apparatus is placed on a table top, is not homogeneous, having a gradient $G=22 \mathrm{~T} / \mathrm{m}$. The signal obtained from an RF coil along the vertical axis is recorded for a very narrow zone in the shape of a flat disk with a thickness of $10 \mu \mathrm{m}$ and a radius of $\pm 2 \mathrm{~cm}$, located a few $\mathrm{cm}$ above the coil. Depth profiles are obtained by moving the block containing the RF coil and the permanent magnets up and down and taking a measurement at each location. In this manner the object can be scanned over a maximum depth of $5 \mathrm{~mm}$. Different experimental settings were chosen to obtain either an optimal signal from the solid materials or from the absorbed water. In the first case a sequence of 32 echoes with echo time $56.2 \mu$ s and a repetition time of 300 ms were used. Each scan was repeated 128 times and the results averaged. Water has a slower relaxation, which was measured with 256 echoes, each $56.2 \mu$ s long, repeated 32 times with a repetition time of $650 \mathrm{~ms}$. The spatial resolution is determined by the step size, which was $40 \mu \mathrm{m}$. For a scan over a sample with thickness 600 $\mu \mathrm{m}$, this leads to a time of approximately 9 minutes to record one profile. 

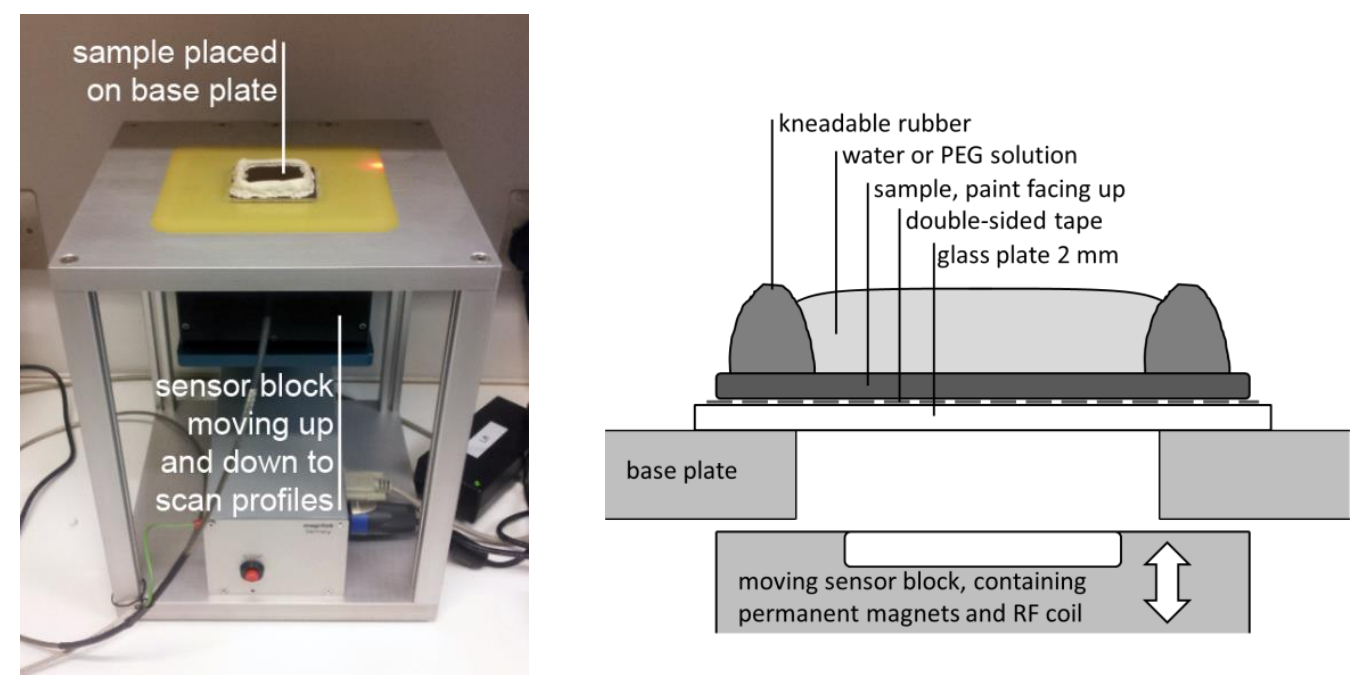

Figure 6. Set-up of the NMR PM5 Mouse: Left: the sample positioned on top of the sensitive volume (which has a square central opening). Right: cross-sectional sketch sample set-up, with painting mock-up affixed on a glass plate with the textile facing down and the paint layer facing up.

\section{GARField NMR set-up}

The GARField (Gradient At Right angle to Field) NMR (Glover, et al., 1999) at Eindhoven University of Technology is an inhouse set-up, making use of electromagnets to create a horizontal $B_{0}$ field, and of parabolic shaped magnets' pole tips able

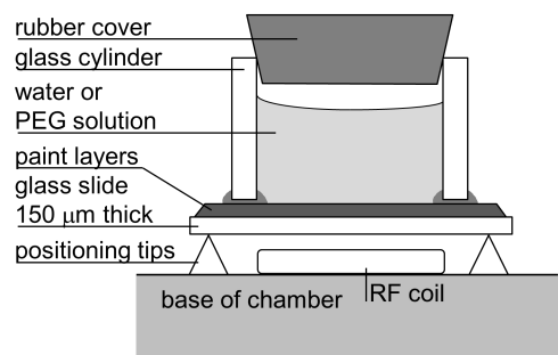


The PEG solutions were chosen because they allow for regulation of the water activity: a higher concentration of PEG results in a lower water activity (Ninni, et al., 1999). High molecular weight PEG was used (PEG 20000, Sigma Aldrich) so as to avoid migration of the polymer moieties into the paint mock-up. PEG solutions were prepared to achieve target water activities of 90 and $95 \%$. The resultant solutions had measured effective activities of 88.4 and $92.7 \%$, respectively. Exposing the sample to a PEG solution of a given water activity corresponds to exposing it to moist air with the same relative humidity, i.e. when the glass cylinder is filled with a PEG solution of $88.4 \%$ activity, the painting mock-up is exposed to conditions equivalent to $88.4 \% \mathrm{RH}$.

The profiles, obtained as signal intensity as a function of depth during water uptake and subsequent drying, were normalised to the profile of a $0.02 \mathrm{M} \mathrm{CuSO}_{4}(\mathrm{aq})$ solution. This processing step allows for the signal intensities to be converted to volume fractions of water (see Reuvers (2012) for details). The added sulphate reduces the relaxation time of the water; otherwise it would be too long to measure all liquid signals with the parameters that are selected for the experiment. The field-of-view showing good signal-to-noise quality is $\sim 400 \mu \mathrm{m}$, which allows for the observation of a 250 $\mu \mathrm{m}$ sample thickness when the thickness of the glass slide is subtracted.

\section{Relaxation experiments on moist canvas monitored with the PM-5 NMR Mouse}

A sample of unsized canvas (type 1) was used to measure the relaxation behaviour in the PM-5 NMR Mouse in ambient conditions, at capillary saturation and during drying. A CPMG sequence consisting of 2048 echoes with a $50 \mu$ s echo time, 16 us acquisition time, and 13000 ms repetition time was used; 128 scans were averaged. An initial measurement was obtained when the sample was in equilibrium with the ambient laboratory condition, i.e. $50+/-5 \%$ RH. The sample was then saturated with water and measurements were carried out during the drying process (1 hour) in laboratory conditions. For comparison, the CPMG relaxation behaviour of a canvas sample fully dried in an oven at $55^{\circ} \mathrm{C}$ for $48 \mathrm{hrs}$ and placed over molecular sieves for an additional $48 \mathrm{hrs}$ was also measured.

\section{Results and discussion}

The neutron radiography results on the reconstructed oil painting and on sized canvas without a paint layer are presented first. Because the canvas is a 3D structure, the distribution of the moisture in the plane of the weave is also shown. Next, the effect of a lining canvas on the moisture absorption behaviour is demonstrated. Lastly, we present the NMR results on the isolated paint layers (GARField set-up) as well as on the painted canvas (Mouse).

\section{Moisture distribution in the oil painting layers and sized canvas}

The dynamics of the moisture absorption and desorption processes in samples 1 and 2 is visualised in Figure 8 . The test procedure consists of $4 \mathrm{hrs}$ of exposure to $90 \% \mathrm{RH}$ followed by $3 \mathrm{hrs}$ of exposure to $15 \% \mathrm{RH}$. It takes less than $30 \mathrm{~min}$ for the samples to surpass $50 \%$ of the final moisture content; after $100 \mathrm{~min}$ the samples have nearly reached saturation. The desorption process occurs faster than the absorption process. Absorption in the canvas is markedly higher than in the ground. 

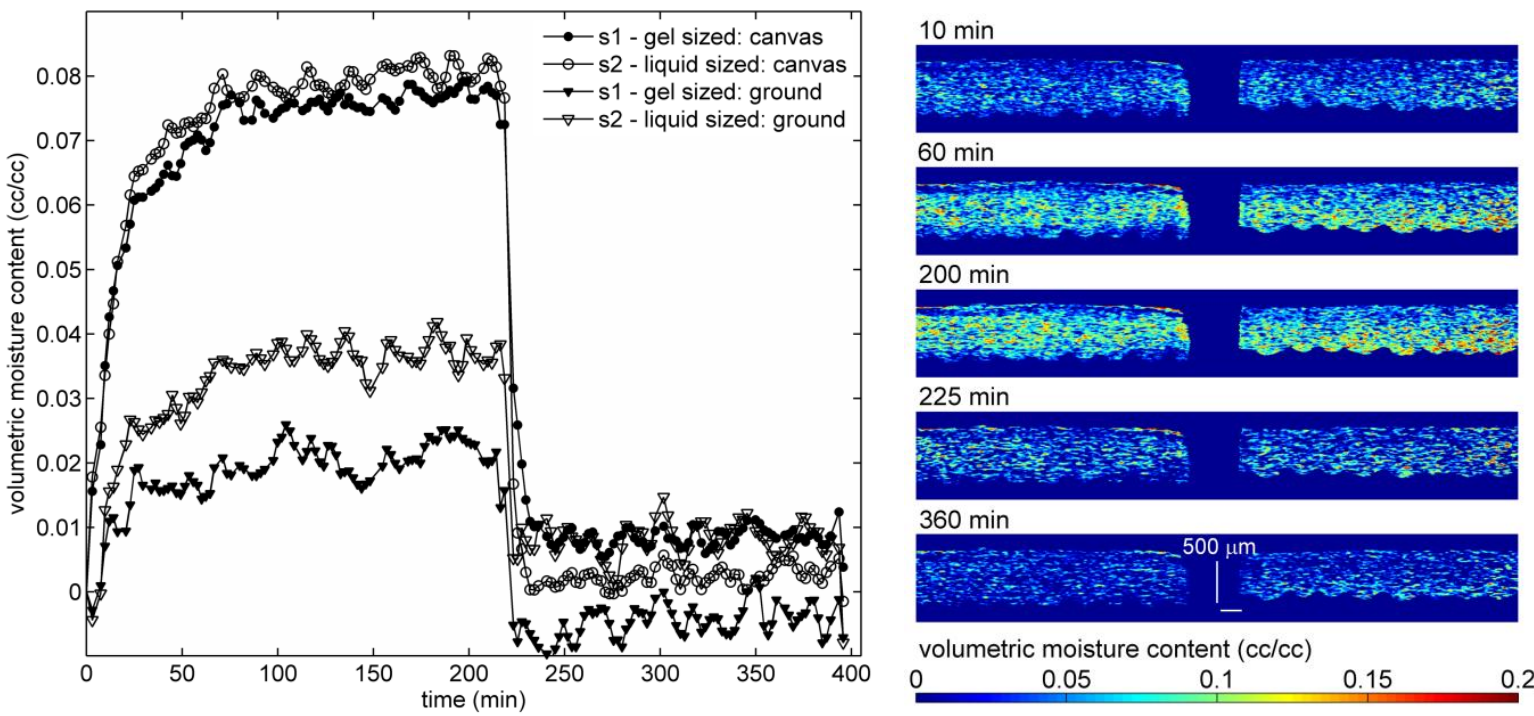

Figure 8. Dynamics of moisture absorption and desorption in sample 1 and sample 2. Left: average moisture content in canvas and ground in both samples versus time. Right: snap-shots of the moisture distribution at 5 chosen points in time. Both scale bars represent $500 \mu \mathrm{m}$ : the image is stretched in the vertical direction.

The differences between sample 1 (having a gel sized canvas) and sample 2 (having a liquid sized canvas) are further clarified by observing the moisture distribution at the end of the 4-hour absorption phase (Figure 9). In this figure, the strong signal at the location of the paint in the gel-sized sample is due to local deformation of the sample rather than high water absorption: the registration procedure described above corrects global deformation and cannot capture local events. When comparing the canvases in both samples, a calculation over a representative area with correction for the slight differences in the depth of the samples shows that the average moisture content is $7 \%$ higher in the liquid-sized canvas than in the gel-sized canvas. In a repeat-experiment using the same samples in a different configuration this difference was $5 \%$. This result can be attributed to the larger glue content in liquid-sized canvas relative to gel-sized canvas: application of the glue as a liquid leads to $\sim 4 \%$ (relative to the mass of the dry canvas) more glue absorption than application of the glue as a gel. By contrast, a gel size penetrates less into the canvas, and stays close to its surface (Hendrickx, et al., 2016). Moreover, due to capillary forces, the canvas pulls together during the application of the liquid glue. The resulting difference in thickness can be observed with the eye. The second, more pronounced difference is observed in the average moisture content of the ground. The ground in the liquid-sized painting absorbs on average $85 \%$ more moisture than the ground in the gel-sized painting. In the repeat-experiment this difference was $30 \%$.

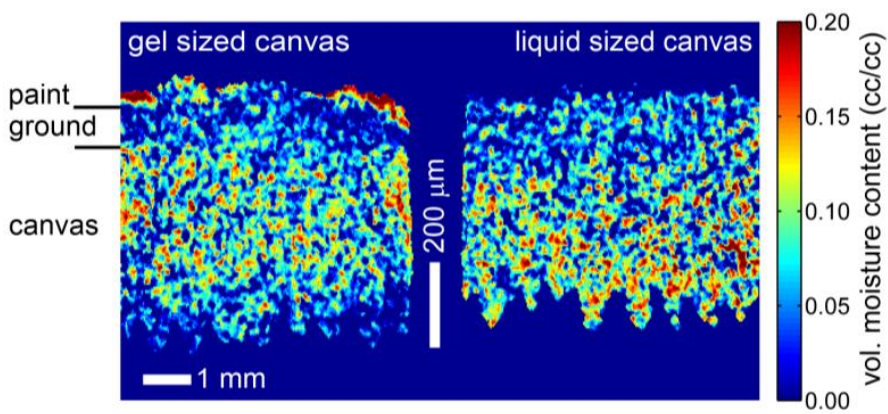

Figure 9. Moisture distribution, expressed as average volumetric moisture content over the depth of the sample, measured with neutron radiography (session 2014). The left image shows sample 1, a gel sized canvas, chalk-glue ground and oil paint; the right image shows sample 2, a liquid sized canvas, chalk-glue ground and oil paint. Due to the use of a tilted detector, the image is stretched in the vertical direction.

The role of glue sizing in the moisture absorption of a painted canvas has been analysed further. During the experimental campaign of 2013, using set-up A, two samples were measured which were half-sized and half-unsized so that exactly the 
same conditions were applied to both types of sample simultaneously (samples 5 and 6, Table 1), and subtle differences could be observed more effectively. For both tests, the moisture profiles after 4 hrs of exposure to high RH are plotted in Figure 10. The curves are averages over bands 150 pixels wide. Noise was reduced by taking the trimmed mean of 5 curves around the target time of $4 \mathrm{hrs}$ (in effect: from 3:57 till 4:03). It should be noted that all profiles in this section and the following are plotted with the bottom of the sample to the left of the image and the top (in this case, the sized surface of the canvas) to the right. In Figure 10 (left) it is clear that the liquid-sized portion of the sample became more compact and absorbed more than the unsized portion of the sample (particularly in the area corresponding to the bottom of the canvas), likely a result of the presence of glue. The gel-sized canvas presents a different response (Figure 10 right): the increase due to the presence of glue is concentrated at the top of the canvas.
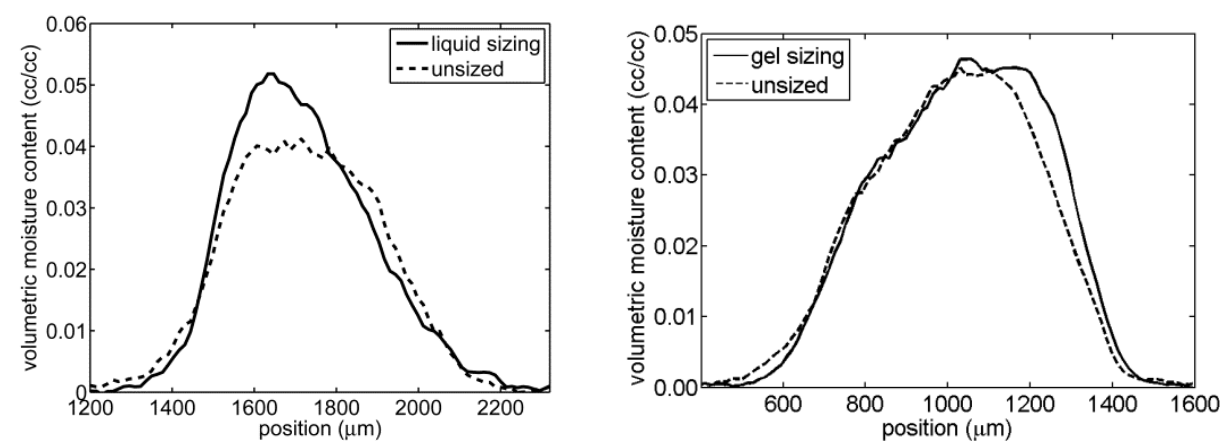

Figure 10. Moisture profiles over unsized and sized canvas after 4 hrs of exposure to high RH, measured by neutron radiography. Left: liquid-sized canvas and unsized canvas. Right: gel-sized canvas and unsized canvas.

The sized canvas sample prepared with open weave canvas (sample 7, Table 1) shows another type of response due to its characteristic weave structure. The openings between the yarns are bridged by a thin transparent film of glue size. The sample used was produced in the framework of a programme aiming to reconstruct Van Gogh's painting technique (Carlyle, L., 2013). The semi-quantitative results of $2.5 \mathrm{hrs}$ of absorption when placed in high RH are plotted in the form of moisture profiles in Figure 11. Because the effective attenuation coefficient is not available for this test, the plotted quantity is a dimensionless value, which is proportional to the moisture content. The curves represent equal time steps of $7 \mathrm{~min}$ and $42 \mathrm{~s}$ and are each the trimmed average of 5 subsequently recorded profiles.

The presence of the accessible glue size film leads to a high absorption at the top of the sample in the initial stage of the wetting process. The peak at the right of the signal profile, centred at $525 \mu \mathrm{m}$ and visible in the initial profiles, is overtaken by the amount of moisture absorbed by the canvas, and is visible as a shoulder in the final profiles (compare to Figure 10 right). This fast and significant uptake of moisture by the glue-film in open-weave canvas is likely due to the large amount of highly-accessible glue present within the open structure (relative to other types of size discussed above). 


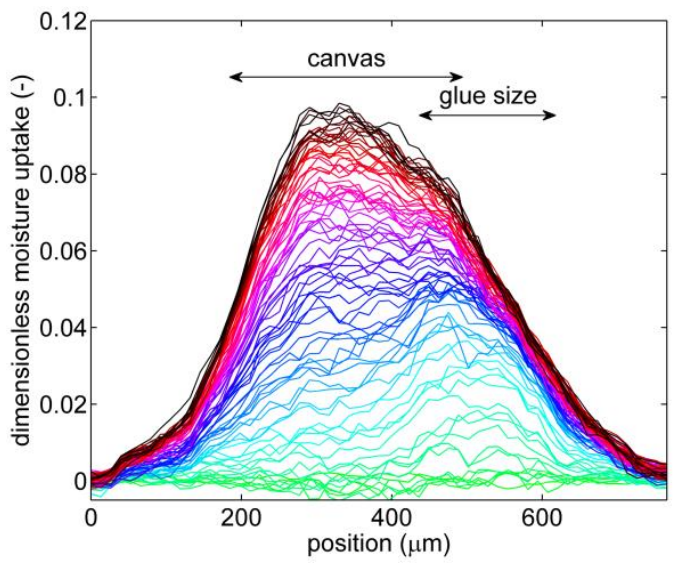

Figure 11. Dimensionless moisture uptake profiles of an open-weave canvas with gel sizing during 2.5 hrs of exposure to high RH. The colours of the curves represent the evolution in time, going from green over to blue, to red and black.

Having established that the linen canvas is, in all cases, and certainly below $95 \% \mathrm{RH}$, the component which absorbs the highest proportion of moisture, it is expected that the distribution of moisture in the plane of a painting approximately matches the weave-pattern. Figure 12 clearly confirms this hypothesis; almost no moisture absorption occurs in the gaps of the weave, where only glue and paint are present.

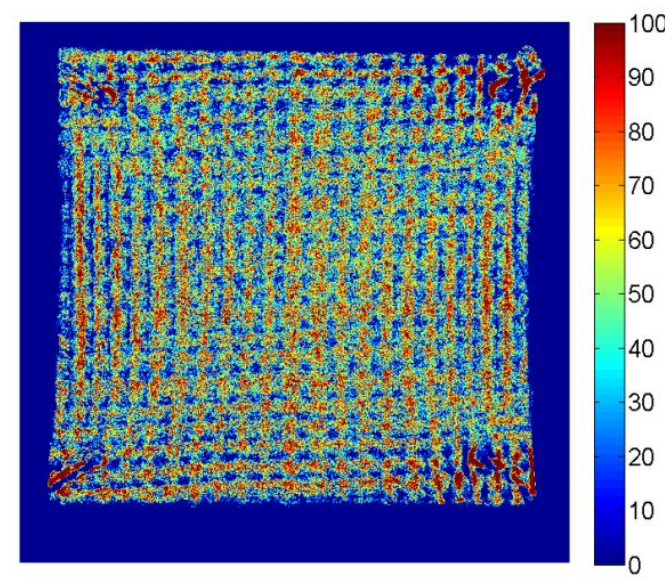

Figure 12. Neutron radiography image of the moisture distribution over a reconstructed painting (sample type 1) in frontal position, expressed in $\mu \mathrm{m}$ of moisture. The thickness of the sample is $\sim 500 \mu \mathrm{m}$. Thus, a value of 50 can correspond to a volumetric moisture content of $10 \%$.

\section{Moisture distribution in a lined painting}

The aim of this section is to explore the extent to which the added BEVA371 ${ }^{\circledR}$ glue film and lining canvas lead to a delay and/or a reduction in the absorption and desorption of moisture undergone by the original painting. Experiments were performed using set-up B, in which samples are exposed to high $\mathrm{RH}$ only from the bottom, while the top is covered with a solid plate. This configuration was conceived of in order to focus on the effect of the lining.

In Figure 13 the final moisture uptake profiles of duplicate tests on linen-lined and polyester-lined canvases are shown. In the linen-lined sample (Figure 13 left) it is apparent that the lining canvas, which is directly exposed to high $\mathrm{RH}$, has absorbed more water than the original canvas. However, in contrast to the BEVA371 ${ }^{\circledR}$ glue-film, the original canvas also absorbs a significant amount of moisture. The results suggest that the lining canvas and glue add an extra vapour resistance on the back of the painting, but by no means form an impermeable barrier. In contrast to the linen lining canvas, the polyester lining canvas absorbs little moisture (Figure 13 right). This result was expected, as polyester fibres are not hygroscopic. The amount absorbed by the original canvas in the polyester-lined sample is comparable to the amount 


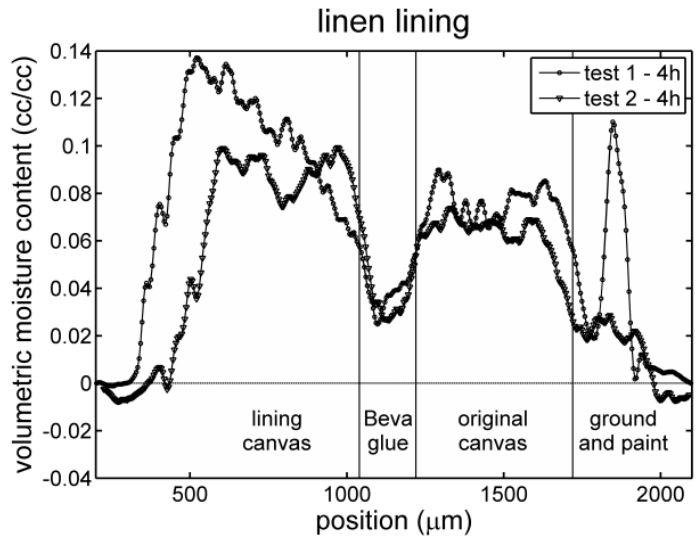

polyester lining

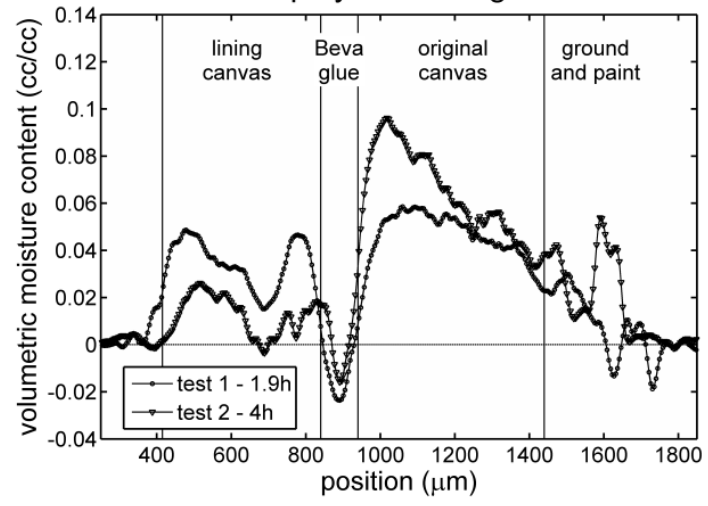

Figure 13. Final moisture profiles over the thickness of lined reconstructed paintings after 4 hrs ( 1.9 hrs in one case) of exposure to $90 \%$ RH; (left) linen-lined, and (right) polyester-lined canvas.

absorbed in by the linen-lined sample; however, the profiles of the former are more skewed to the left. The moisture content at the position of the ground and paint layers has a baseline at low moisture content, but shows high peaks in two out of four curves. As mentioned in the discussion of the results for the unlined painting, these non-systematic high values are experimental features which occur on the sample edges due to complex deformations of the samples upon absorption, which could not be compensated by the registration procedure. They are not to be interpreted as real actual high moisture contents.

In Figure 14, the average moisture content in the centre of the original linen canvas (for the two types of lined samples) is plotted over time, and compared with data from the same location in the unlined canvas (i.e. the left sample in Figure 9). From the graph, it is apparent that the lining acts as a damper to moisture uptake, so that the reaction of the painting to ambient moisture fluctuations is slowed. After $1 \mathrm{hr}$ of high-RH exposure, the moisture content is only one-third of the amount in the unlined canvas; after $4 \mathrm{hrs}$, this value has reached $~ 80 \%$. The delay in response is even more pronounced in the drying stage. The residual moisture content after $3 \mathrm{hrs}$ of drying is still half of the initial value, while it has dropped to $\sim 10 \%$ in the unlined painting. The difference between the two types of lining is not significant.

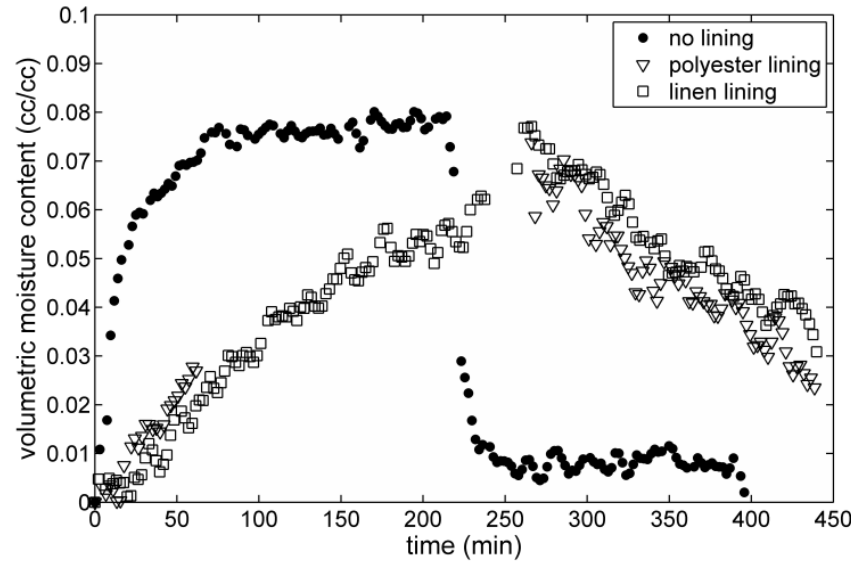

Figure 14. Comparison of the absorption and desorption in the centre of the linen canvas of an original painting in three cases: the unlined painting, polyester-lined, and linen-lined. The dataset of the polyester-lined sample is incomplete due to beamline downtime; the dataset of the linen-lined sample is composed of two separate sets for wetting and drying.

The key conclusions from the observation of lined samples can be summarised as: (1) the BEVA371 ${ }^{\circledR}$ glue film absorbs little moisture and does not form a vapour barrier between the original and the added canvas material as the moisture still reaches the original canvas; (2) nevertheless the increase in vapour resistance due to the lining is significant and comparable for linen and polyester lining; (3) the polyester lining absorbs less than the linen lining due to its lower 
hygroscopicity; and (4) lining has a marked retarding effect on the moisture response of the original painting, which is more pronounced during desorption than during absorption.

\section{Moisture absorption through the paint layers measured by NMR Mouse}

The diffusion of water into and through the oil paint layer in the reconstructed oil painting samples was measured by NMR Mouse during continuous exposure to water and PEG solutions. Sorption in contact with water can be compared to exposure of the painting to water vapour with $\mathrm{RH}=100 \%$. Using PEG solutions, the activity of water is lowered and the sorption characteristics correspond to sorption from vapour with an $\mathrm{RH}$ of $92.7 \%$. Figure 15 shows the moisture absorption profiles, and the consequent drying profiles of such an NMR experiment. Quantities are averaged signal intensities over the first 64 echoes and they are normalised, i.e. divided by the signal strength of a $0.02 \mathrm{M} \mathrm{CuSO}_{4}$ solution as described in the methods section, so that they can be interpreted as volume fractions of water. The steep increase of the signal on the right side (top of the sample) in the absorption profiles corresponds to the edge of the water column on top of the sample, and does not reflect absorbed moisture in the sample. It is absent in the drying profiles, where the sample is exposed to lab conditions for $8 \mathrm{hrs}$.

The profiles reflect the uptake in the canvas and the ground, but do not have sufficient spatial resolution to reveal the processes occurring in the oil paint. The bell-shaped curves are in agreement with the findings from neutron radiography experiments. Water saturation appears to occur at volumetric moisture contents between 22 and $25 \%$. This value can be related to the isotherm of the flax and the glue (Figure 1) and corresponds to a mass fraction of 20 to $22 \%$ of the flax dry mass, assuming that $60 \%$ of the canvas volume is occupied by the fibres (in the middle of the canvas), of which the cell wall material has a mass density of $1.45 \mathrm{~g} / \mathrm{cc}$. Knowing that the flax in lab conditions is likely to contain $6-8$ mass\% moisture, the saturated fraction of moisture would be 26 to $230 \%$. This value lies between the expected equilibrium values of flax and glue at $100 \% \mathrm{RH}$, being closer to the value for flax. In similar experiments using PEG solutions with water activities of 92.7 and $88.4 \%$, the same calculation leads to equilibrium moisture contents of 20 to $22 \%$ and 18 to $20 \%$ by mass, respectively. This large difference in absorption over the range between 90 and $100 \%$ RH corresponds to the steep increase of the Sshaped sorption isotherm.
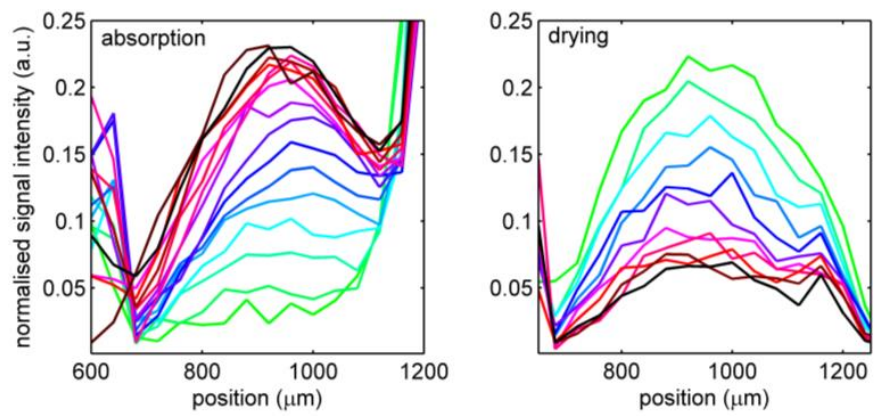

Figure 15. Moisture profiles during absorption (a) and drying (b) of the gel-sized oil painting through the paint layers, obtained with the NMR Mouse. The time step between two subsequent profiles is 41 minutes (final absorption signal recorded after 14.5 hrs).

\section{Moisture distribution over isolated paint layers (ground and oil paint)}

The GARField NMR set-up offers sufficient resolution to measure a number of points inside the oil paint and ground layers. The measured mock-up samples consisted of ground and oil paint only, and were measured first dry, then during absorption of moisture from a column of deionised water or PEG solution on top of the oil paint, and finally during airdrying in lab conditions. Figure 16 shows plots of the dry profiles and wet profiles after $2 \mathrm{hrs}$ of contact with the liquid. The quantity plotted is the signal intensity of the first echo, normalised to the signal intensity of a $\mathrm{CuSO}_{4}$ solution (see methods 
section), allowing for the increase of the curve to be interpreted as a local volumetric fraction of absorbed water. During contact with water (Figure 16 left), the signal increase is significant; $~ 15 \%$ of the sample volume is occupied by water after 2 hrs. However, when the activity of the liquid in contact with the paint is reduced to $93 \%$, less than $2.5 \%$ of the volume of the paint layers is occupied by water in the same time period. Within the paint layers, as was the case for the sized canvas, there appears to be an onset of strong moisture absorption starting at $\mathrm{RH}$ above $93 \%$, reflecting the large increase of the isotherm (Figure 1).
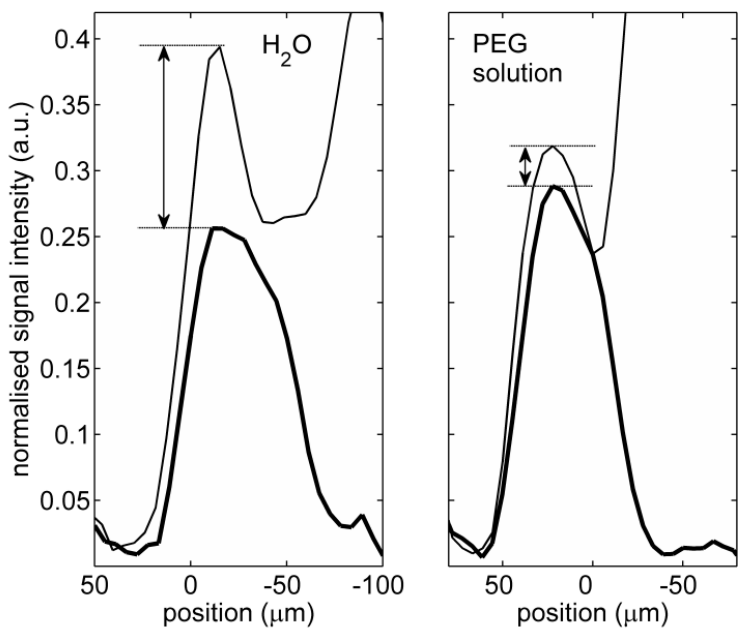

Figure 16. GARField NMR intensity profiles of the ground and oil paint set-up obtained when dry (tick line) and after $\mathbf{2}$ hrs of contact with water (thin line) (left) or a PEG-solution of water activity $93 \%$ (right). The normalised signal intensity corresponds to the volumetric moisture content. The steep increase in signal intensity on the left side of the wetted curves is the edge of the liquid reservoir above the sample.

When exposure to water was maintained for $68 \mathrm{hrs}$, saturation was reached at volumetric moisture contents of up to $50 \%$ (Figure 17). In this saturated state, the surface of the paint was characterised by the local formation of osmotic blisters, which started appearing after 4 to 8 hrs of exposure; the blisters do not seem to affect the shape of the intensity profile curves. This large uptake of moisture is concentrated on the left side of profile, i.e. the location of the ground layer, and reflects the increase of the glue isotherm at high $\mathrm{RH}$.

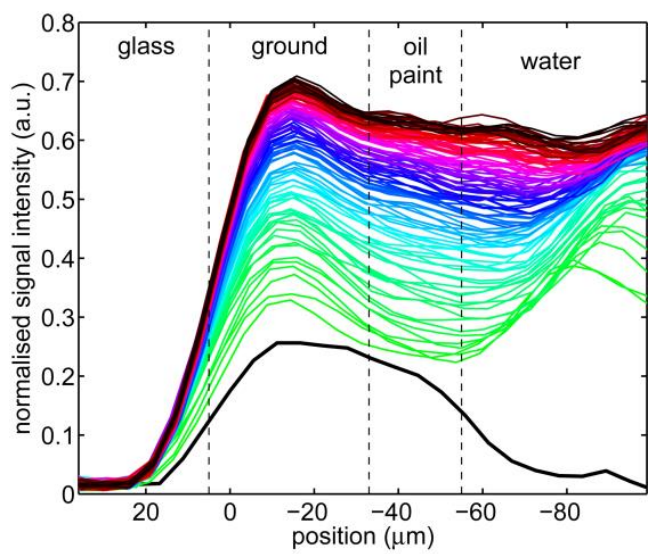

Figure 17. Moisture uptake profiles during a $68 \mathrm{hr}$ absorption experiment on a ground and oil paint sample. The water column is on the right, on top of the oil paint; the ground is on the left.

This large moisture absorption is accompanied by an increase in volume of the two-layer system. It is difficult to distinguish the interface between the sample and water column in Figure 17. The hand-painted sample-films present an irregular surface, resulting in a transition zone at the surface. A good method to monitor the edge of the solid material, and in doing so, quantify the swelling, is to calculate an averaged relaxation time $\left\langle T_{2}\right\rangle$ for each signal point (Steinbrecher et al. 2000, 
Reuvers 2012). The presence of bulk water leads to a strong increase of relaxation time; 1.5 ms was found to be a good threshold value (Figure 18). Figure 19 shows the volume increase of the sample based on this criterion, versus the water uptake calculated as an integral over the profiles. The scatter plot indicates a linear correlation with a slope of $11.2 \mathrm{cc}$ per mole of absorbed water. For comparison, the molar volume of liquid water is $18.04 \mathrm{cc} / \mathrm{mol}$. This result indicates that a large fraction of the water is present within the structure of the polymeric network, causing it to swell, while a smaller but significant portion is filling the pores of the ground.

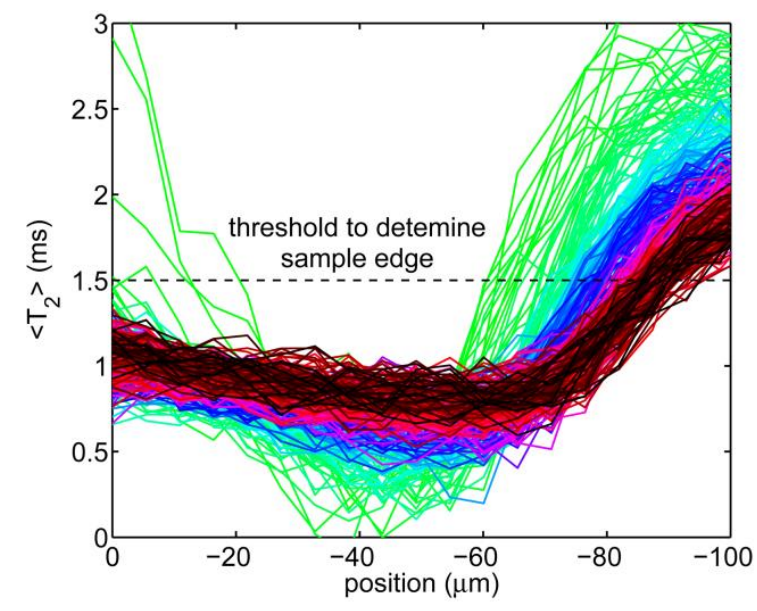

Figure 18. Evolution of the averaged relaxation time $\langle$ T2 $>$ over the position of the sample. The colours of the curves represent the evolution in time, going from green over blue to red and black.

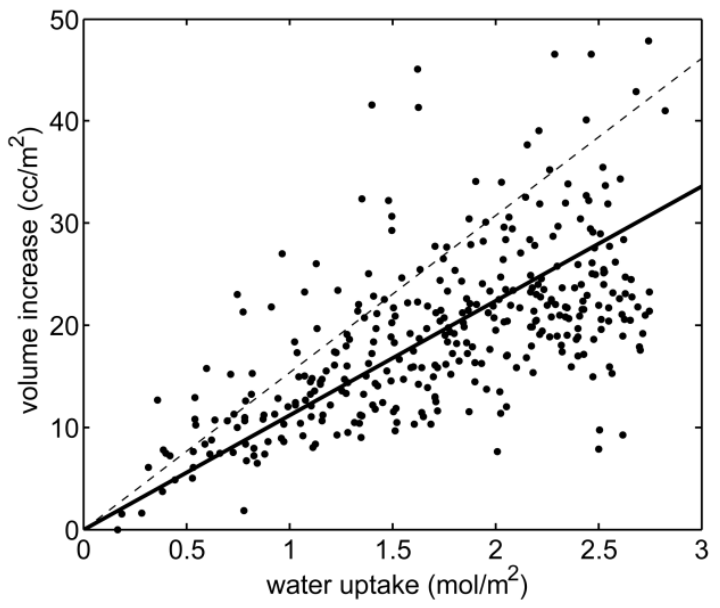

Figure 19. Volume increase of the sample (ground and oil paint) vs the amount of water uptake during a $\mathbf{6 8} \mathrm{hrs}$ absorption experiment. The solid line is the linear regression line; the dashed line corresponds to the molar volume of water.

\section{The state of water in wetted canvas}

The NMR Mouse set-up was also used to obtain information about the state of moisture within the canvas at ca. $50 \% \mathrm{RH}$ laboratory conditions (ambient conditions), during full saturation with water, and a subsequent drying phase. The $T_{2}$ of a fully dried (see Methods section for details), unsized linen canvas was found to be approximately $100 \mu \mathrm{s}$. In ambient laboratory conditions, moisture from the atmosphere is absorbed into the canvas fibres, resulting in the appearance of a second component with a relatively longer $T_{2}$ value between 1 and $3 \mathrm{~ms}$. This component has been interpreted to represent the bound water present within the flax fibres. Upon full wetting of the canvas sample, a third component with a much longer $T_{2}$ is detected, with a value fluctuating between 12 and $19 \mathrm{~ms}$; this component is the bulk unbound water (or 'free' water) between the canvas fibres in the water-saturated canvas. By monitoring the canvas as it dries, the unbound water $T_{2}$ and its amplitude are found to decrease, while the amplitude and relaxation time of the bound water is found to remain relatively stable (Figure 20 ).
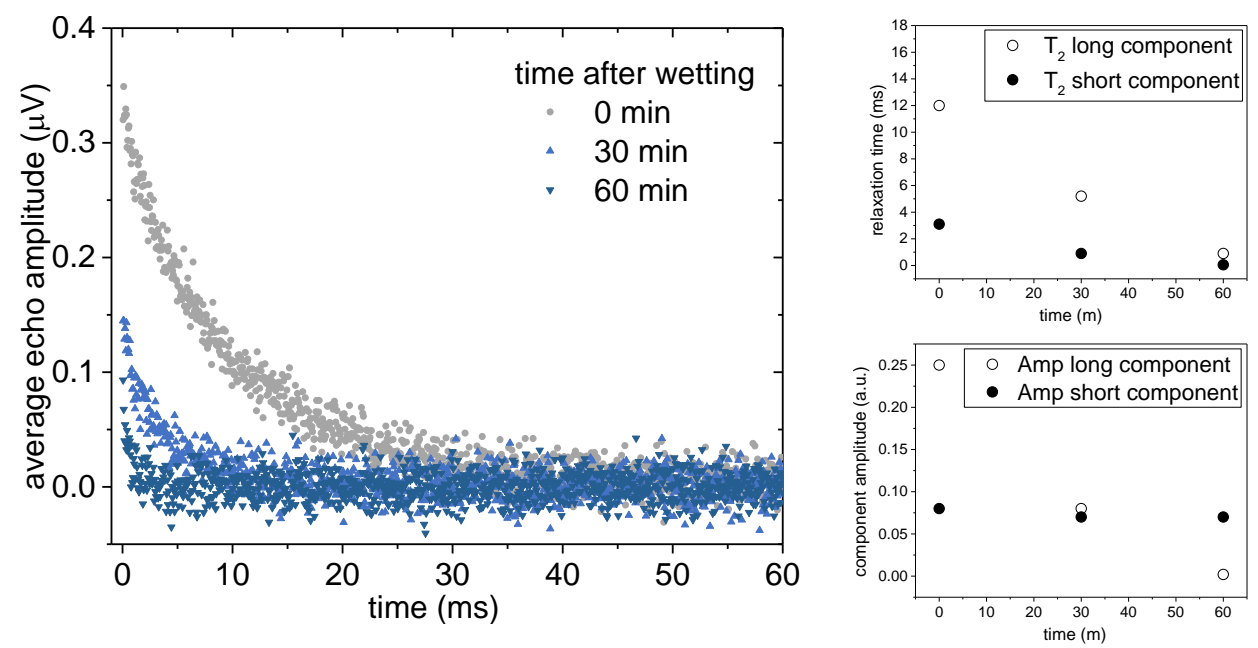
Figure 20. Left: NMR MOUSE measurements of CPMG relaxation curves for the moist canvas sample during drying at ambient conditions. Right top: $\mathrm{T}_{2}$ of the short (canvas) and long (bound water) components during the drying period monitored; right bottom: amplitudes of the two components over the same time-period; the $T_{2}$ and amplitude values were obtained by fitting the relaxation curves with a biexponential decay.

\section{Conclusions}

This paper presents how state-of-the-art neutron radiography and NMR can be used for the spatially and temporally resolved visualisation and quantification of moisture in paintings. The results, which can be represented as series of moisture profiles, sequences of 2D colour plots, curves of volume-averaged moisture content, or animated graphics, capture the complexity of the dynamics of moisture uptake and drying in an unambiguous and understandable way. Neutron radiography is a versatile technique for measuring the spatial distribution of moisture in painting samples and allows visualising features as small as a few tens of micrometres. The resolution in one direction can be increased by a factor 2.88 ( 5.76 divided by 2 due to binning) by using a tilted detector.

However, when doing so, two factors become critical: accurate referencing to address the sample swelling and deformation, and decreasing lower signal-to-noise ratio. The obtained results provide a clear image of the moisture absorption and desorption in the canvas and the ground during the imposed experimental procedures of steps in $\mathrm{RH}$ from dry to $90 \%$ and back to $15 \%$. As can be seen in the isotherms, collagen glue absorbs the highest amount of moisture per weight. It is therefore understandable that the application of a small amount of glue size on the canvas gives rise to a measurable difference in water sorption properties; a local moisture surplus at the location of the glue was found. In the case where the glue was highly accessible to moisture, it was also found to absorb faster than the textile. The dominant role of the textile canvas in the absorption process was demonstrated by changing the sample orientation: the moisture content between the yarns is much lower than within the yarns. In general the speed of absorption is high: in less than 100 min of exposure to $93 \% \mathrm{RH}$, the sample had reached saturation.

The effect of a lining on the absorption and desorption of moisture in a painting was studied for the case of a BEVA $371^{\circledR}$ adhesion film and two types of canvas: linen and polyester. The adhesive film absorbs little moisture within the measured timespan. The absorption of the original painting is strongly delayed in the samples with either type of lining; moisture content otherwise reached in 10 minutes (4\%) in unlined samples, is only reached after 100 minutes in the lined samples. The damping effect is even stronger during desorption. In most cases the buffering effect of the lining will be evaluated as positive for the conservation of the original painting, but we have to note that this does not take into account hygromechanical considerations. The lining canvas swells and shrinks as well, having similar or different properties to the original one, and stresses can be transferred to the original canvas via the glue layer. Note that other historically relevant methods of lining might result in a very different behaviour.

The difficulties in monitoring moisture uptake in the relatively thin ground and paint layers were overcome through NMR experiments. The best spatial resolution, down to $5 \mu \mathrm{m}$, was attained in the GARField experiments. By using PEG solutions, we could explore moisture uptake in the range of $\mathrm{RH}$ between 90 and $100 \%$, which is difficult to control with conditioned air. When in contact with a pure water, both ground and paint layer absorb over 3 times more than in case of exposure to a solution of water activity $93 \%$. When the experiment is continued until saturation, the hydrophilic nature of the glue leads to moisture content levels of up to $50 \%$. Quantifying the swelling of layers based on the signal intensity profiles is not straightforward because of the uneven interface between the water reservoir and paint surface. However, it was possible to use the relaxation behaviour of the paint and water as a suitable criterion for the location of the interface, and by doing so to calculate the swelling. 
The NMR Mouse was used to monitor the ingress of moisture through the paint layer, which is the least permeable in the build-up of the painting. A prolonged contact with liquid is not representative of common practice, but it enables the derivation of parameters such as the saturation moisture content of the layers and the permeability of the top layer. One practical application of these parameters is that we may be able to predict how much water will diffuse in the painting when an aqueous cleaning gel with a certain water activity remains on the surface during a fixed amount of time.

The results of the NMR Mouse experiments using PEG solutions with different activities, as well as those of the GARField set-up and of the neutron radiography, are in good quantitative agreement with the sorption isotherms of the materials. Whereas the neutron radiography experiments allowed for an RH of up to $93 \%$ in the climatic chamber, NMR allowed for an insight into the processes taking place at water activities at higher $\mathrm{RH}$, the range corresponding to the steep increase of water uptake in the isotherms. Also the high water uptake of the glue in the ground layer, which only appears in the isotherm above $95 \% \mathrm{RH}$, could clearly be observed in the NMR studies. Furthermore NMR can be used to discriminate quantitatively between the fractions of bound and unbound moisture in a canvas as a function of space and time. This ability can be useful in conservation practice: for treatments such as flattening deformed paintings, one would typically need bound moisture and not free water, in order to make the deformed materials more flexible. A setup can be imagined where the NMR Mouse is used as an on-line tool in the workshop to monitor (and help optimise) the wetting process.

The methods presented in this paper can further be developed to include advanced observations on the hygro-mechanics of paintings, especially using neutron tomography. 3D-Computed (neutron or X-ray) tomography, can be complementary, in that it captures the combination of dilatation and warping which occurs when a sample is freely deforming; however, these experiments will necessarily suffer from a low temporal resolution and the difficulty of a straightforward registration. Such studies will create the possibility to make a link to the considerable corpus of experimental data published on the hygromechanics of canvas paintings during the last decades (Mecklenburg \& Tumosa, 1991; Young, 1996). At present, we have mainly focused on the moisture distribution in the painting layers; deformation was an experimental issue rather than a primary point of attention. Preliminary tests (not reported here) have revealed a complexity, which needs further experimental and analytical efforts to be elucidated. It is to be expected that 3D numerical models will provide better possibilities for understanding the experimental results. Complementing the dataset to types of paint different from the ones used for this investigation is necessary to broaden the applicability of the approach.

\section{Acknowledgements}

We are grateful to the technical staff at EMPA Empa Dübendorf, and FOM-Atomic and molecular Institute AMOLF Amsterdam for the design and production of the experimental set-ups, especially to Stefan Carl and Beat Margelisch. Jan Hovind and Pierre Boillat supported the work in the ICON beamline. Eleanor Cato assisted during the 2014 session. Vladimir Vilde provided useful comparative results obtained with the NMR Mouse at UCL. The technical staff at the Department of Applied Physics, Technical University of Eindhoven, kindly assisted where needed during the GARField NMR sessions. Most of the samples were produced at SIK-ISEA with the help of the restoration workshop's staff: Danièle Gros, Markus Küffner, Margaux Genton, and Karoline Beltinger. One of the samples was kindly provided by Leslie Carlyle. Finally, the Werner Abegg Foundation is acknowledged for providing the necessary funds for this work. Lora Angelova would like to thank the Royal Society for funding the NMR Mouse research through a Newton Fellowship. 


\section{References}

Baukh, V., Huinink, H.P., Adan, O.C., Erich, S.J., van der Ven, L.G., 2010. NMR imaging of water uptake in multilayer polymeric films: stressing the role of mechanical stress. Macromolecules, 43: 3882-3889.

Baukh, V., Huinink, H.P., Adan, O.C.G., Erich, S.J.F., van der Ven, L.G.J., 2012. Predicting water transport in multilayer coatings. Polymer, 53: 3304-3312.

Beltinger, K., Ferreira, E.S.B., Wyss, K., 2015. Kunsttechnologische Forschungen zur Malerei von Cuno Amiet (1883-1914), KUNSTmaterial. Scheidegger \& Spiess: Zürich.

Berger, G.A., 1972. Formulating adhesives for the conservation of paintings, in: Conservation of Paintings and the Graphic Arts, Lisbon Congress, 1972 IIC. London, pp. 613-629.

Bertolin, C., Camuffo, D., 2015. Past reconstruction and future forecast of domains of indoor relative humidity fluctuations calculated according to EN15757:2010. Energy and Buildings, 102: 197-206.

Blau, B., Clausen, K.N., Gvasaliya, S., Janoschek, M., Janssen, S., Keller, L., Roessli, B., Schefer, J., Tregenna-Piggott, P., Wagner, W., others, 2009. The Swiss Spallation Neutron Source SINQ at Paul Scherrer Institut.

Blümich, B., Perlo, J., Casanova, F., 2008. Mobile single-sided NMR. Progress in Nuclear Magnetic Resonance Spectroscopy, 52: 197-269.

Boillat, P., Frei, G., Lehmann, E.H., Scherer, G.G., Wokaun, A., 2010. Neutron imaging resolution improvements optimized for fuel cell applications. Electrochemical and Solid-State Letters, 13: B25-B27.

Boon, J.J., Hendrickx, R., Eijkel, G., Cerjak, I., Kaestner, A., Ferreira, E.S.B., 2015. Neutron radiography for the study of water uptake in painting canvases and preparation layers. Applied Physics A - Materials Science and Processing, 121: 837-847.

Brophy, S.S., Wylie, E., 2013. The Green Museum: A Primer on Environmental Practice. Lanham: AltaMira Press.

Capitani, D., Di Tullio, V., 2012. Nuclear magnetic resonance to characterize and monitor Cultural Heritage. Progress in Nuclear Magnetic Resonance Spectroscopy, 64: 29-69.

Carlyle, L., 2013. De Mayerne Programme - HART Report. Unpublished report. Canadian Conservation Institute (CCI), The Netherlands Institute of Cultural Heritage (ICN) and The Netherlands Institute for Atomic and Molecular Physics (FOMAMOLF).

Carlyle, L., 2012. Exploring the grammar of oil paint through the use of historically accurate reconstructions, In: J.H. Stoner and R. Rushfield, eds. Conservation of Easel Paintings. London: Routledge, pp. 35-38.

Ferreira, E.S.B., Boon, J.J., Stampanoni, M., Marone, F., 2011. Study of the mechanism of formation of calcium soaps in an early 20th century easel painting with correlative 2D and 3D microscopy, in: Preprints of 16th Triennial ICOM-CC Conference, Lisbon: ICOM-CC. pp. 1604-1608.

Glover, P.M., Aptaker, P.S., Bowler, J.R., Ciampi, E., McDonald, P.J., 1999. A novel high-gradient permanent magnet for the profiling of planar films and coatings. J. Magn. Reson. 139, 90-97.

Hackney, S., Reifsnyder, J., te Marvelde, M., Scharff, M., 2012. Lining easel paintings, In: J.H. Stoner and R. Rushfield, eds. Conservation of Easel Paintings. London: Routledge, pp. 415-452.

Hedley, G., 1993. The practicalities of the interaction of moisture with oil paintings on canvas, in: C. Villers, ed., Measured Opinions: Collected Papers on the Conservation of Paintings. London: United Kingdom Institute for Conservation, pp. 112122.

Hendrickx, R., Desmarais, G., Weder, M., Ferreira, E.S.B., Derome, D., 2016. Moisture uptake and permeability of canvas paintings and their components. Journal of Cultural Heritage, available online.

Huijbregts, Z., Kramer, R.P., Martens, M.H.J., van Schijndel, A.W.M., Schellen, H.L., 2012. A proposed method to assess the damage risk of future climate change to museum objects in historic buildings. Building and Environment, 55: 43-56.

Kaestner, A.P., Hartmann, S., Kühne, G., Frei, G., Grünzweig, C., Josic, L., Schmid, F., Lehmann, E.H., 2011. The icon beamline-a facility for cold neutron imaging at SINQ. Nuclear Instruments and Methods in Physics Research Section A: Accelerators, Spectrometers, Detectors and Associated Equipment, 659: 387-393.

Mecklenburg, M.F., 2007. Determining the Acceptable Ranges of Relative Humidity And Temperature in Museums and Galleries. Part 1. Structural response to Relative Humidity. Maryland: Smithsonian Museum Conservation Institute.

Mecklenburg, M.F., Tumosa, C.S., 1991. Mechanical Behavior of Paintings Subjected to Changes in Temperature and Relative Humidity. Washington: National Gallery of Art. 
Michalski, S., 2007. The ideal climate, risk management, the ASHRAE chapter, proofed fluctuations, and toward a full risk analysis model. Experts roundtable on sustainable climate management strategies, Los Angeles: Getty Conservation Institute, 19 pages.

Michalski, S., 1991. Paintings, their response to temperature, relative humidity, shock and vibration. In: M.F. Mecklenburg, ed., Art in Transit: Studies in the Transport of Paintings, Washington: National Gallery of Art, pp. 223-49.

Ninni, L., Camargo, M.S., Meirelles, A.J.A., 1999. Water activity in poly (ethylene glycol) aqueous solutions. Thermochimica Acta, 328: 169-176.

Ollion, J., Cochennec, J., Loll, F., Escudé, C., Boudier, T., 2013. TANGO: a generic tool for high-throughput 3D image analysis for studying nuclear organization. IEEE Transactions on Image Processing, 7: 27-41.

Ordonez E., Twilley J., 1998. Clarifying the Haze, Efflorescence on Works of Art. Analytical Chemistry, 69: 416A-422A.

Perfect, E., Cheng, C.-L., Kang, M., Bilheux, H.Z., Lamanna, J.M., Gragg, M.J., Wright, D.M., 2014. Neutron imaging of hydrogen-rich fluids in geomaterials and engineered porous media: A review. Earth-Science Reviews, 129: $120-135$.

Reuvers, J.W.R., 2012. Water and ion transport in nylon as studied by NMR. PhD thesis. Eindhoven University of Technology.

Roels, S., Carmeliet, J., 2006. Analysis of moisture flow in porous materials using microfocus X-ray radiography. International Journal of Heat and Mass Transfer, 49: 4762-4772.

Steinbrecher, G., Scorei, R., Cimpoiasu, V.M., Petrisor, I., 2000. Stable reconstruction of the T 2 distribution by lowresolution NMR measurements and the classical Markov and Hausdorf momentum problem. Journal of Magnetic Resonance, 146: 321-334.

Thévenaz, P., Ruttimann, U.E., Unser, M., 1998. A pyramid approach to subpixel registration based on intensity. IEEE Transactions on Image Processing, 7: 27-41.

Van Loon, A., Noble, P., Burnstock, A., 2012. Ageing and deterioration of traditional oil and tempera paintings. In: J.H. Stoner and R. Rushfield, eds. Conservation of Easel Paintings. London: Routledge, pp. 214-241.

Young, C., Jardine, S., 2012. Fabrics for the twenty-first century: As artist canvas and for the structural reinforcement of easel paintings on canvas. Studies in Conservation, 57: 237-253.

Young, C.R., 1996. Measurement of the biaxial tensile properties of paintings on canvas. PhD thesis. University of London. 